\title{
Research on Adaptive Control System of Robot Arm with Six Degrees of Freedom
}

\author{
Changgeng $\mathrm{Yu}^{1, \mathrm{a}}$ and Suyun $\mathrm{Li}^{1, \mathrm{~b},{ }^{*}}$ \\ ${ }^{1}$ School of Mechanical and Electronic Engineering, Hezhou University, \\ No.18, xihuan Road, Hezhou, China \\ ayuchanggen66@163.com, bsyxwx@126.com \\ *The corresponding author
}

Keywords: Robot arm with 6 degrees of freedom; Adaptive control; Arduino platform

\begin{abstract}
This paper aims to exploit the adaptive control robot arm with six degrees of freedom, an adaptive fuzzy control system was established. a fuzzy controller, the robot arm trajectory planning and the D-H algorithm were studied based on the fuzzy controller. Firstly, fuzzy controller and control rules are designed according to a series of characteristics of fuzzy control method. Then, based on the analysis of robot arm parameters, robot arm trajectory plan by the D-H algorithm. Finally, Hardware and software design and experiment were complete. Experimental results show that the D-H algorithm can be used to establish the model of the robot arm, and the MATLAB can be used to understand the data processing. The stability of adaptive control of robot arm with 6 degrees of freedom is good, the expansibility is strong, easy to control and so on.
\end{abstract}

\section{Introduction}

Most robot tasks require that robot arms interact with the different objects in the work environment because of savings, quality, safety, and production [1-6]. Several different control methods (i.e., neural network based adaptive control, robust based adaptive control, model reference based adaptive control, and classical PID control) are used to analyze the control performance. The neural network based adaptive control method has strong self-learning, highly nonlinear, and memory function etc. It shows its superiority in solving highly nonlinear problems, and provides a new method for the research of nonlinear control. But it is still in the exploratory stage, it has not formed a relatively complete theoretical and systematic design. The robust based adaptive control has strong self-learning and adaptive control characteristics, particularly in dealing with some external uncertain factors with the good robustness and stability. But in the initial stage of the system operation, there may be a larger tracking error or system delay. The model reference based adaptive control method to obtain and adaptive law is easy to realize, guarantee a fast global stability, adaptive velocity. However, when the robot model changes with the state variables, it cannot be implemented in real time to control the robot. The classic PID control algorithm is simple in principle, easy to use, the control quality of the object change is not too sensitive. However, because the parameters are difficult to be adjusted, the PID algorithm is not conducive to the use of where the environment is changing.

For problems in the above control method, in this paper put forward based on fuzzy adaptive control method, it has good adaptive, robust and stability. The advantages and modeling method is simple, does not need to build a complex mathematical model. The controller and the control rules can be changed very well when was changed environment, which is very suitable for the adaptive control of the robot under harsh environment.

\section{Model of Robot Arm Control System Based on Adaptive Fuzzy Control}

Self-adaptive Fuzzy Control Principle. Fuzzy control is a intelligent control method which based on fuzzy set theory, fuzzy linguistic variables and fuzzy logic, it is an intelligent control method which 
the imitation of fuzzy reasoning and decision making process. The schematic diagram of the fuzzy control system is shown in Fig. 1.

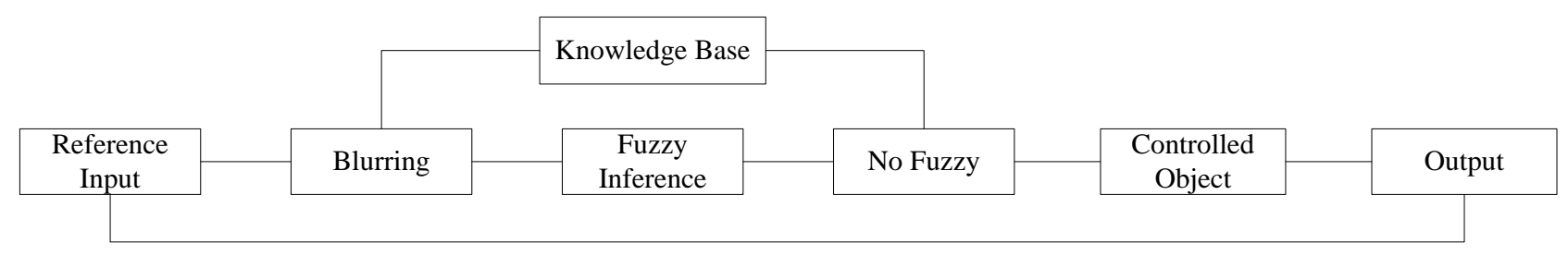

Figure 1. Schematic Diagram of Fuzzy Control System

From Fig. 1 we can shown that fuzzy control method is a kind of effective method for processing imprecise and uncertain problems in inference and control system, and an important part of intelligent control.

Main features are: fuzzy control only requires the operator or expert of experience, knowledge and manipulate data, Don't need to establish the mathematical model of the process, so the application is not easy to obtain accurate mathematical model of the controlled process, structure and parameters is not very clear. The fuzzy control is a kind of language variable controller, the control rule using linguistic variables in the form of qualitative expression, the transfer function and the state equation, controller design methods to summarize the experience of people, from extracts rules, gives direct language variables, and application of reasoning method was used to observe and control. The robust control, especially for nonlinear and time-varying, delay system. From a different point of view, we can design different objective function, the linguistic control rules are independent, but design of the whole system can get the overall coordinated control.

(1) Design of Fuzzy Controller. Based on the characteristics of the fuzzy control, the corresponding fuzzy controller is designed for the control system of the robot arm. Fig. 2 is a block diagram of fuzzy controller.

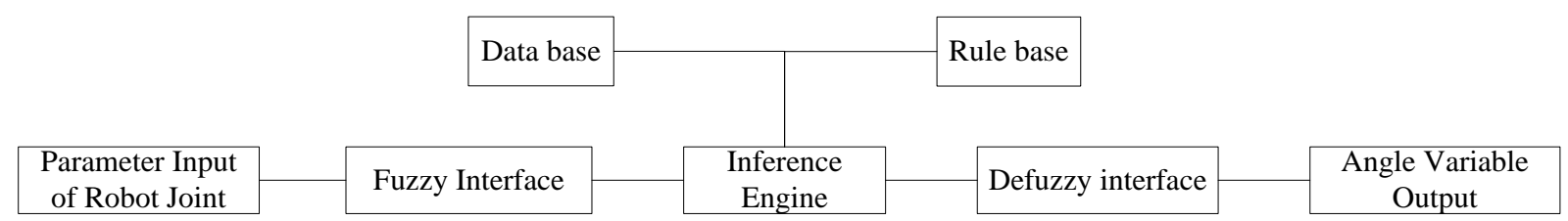

Figure 2. Block Diagram of Fuzzy Controller

A control strategy using fuzzy IF-THEN rules can have the good control performance [7-8]. Fuzzy control design rules as below.

(1)if $\mathrm{A}$ and $\mathrm{B}$ is ... then $\mathrm{C}$ is...; (2)if $\mathrm{A}$ or $\mathrm{B}$ is ... then $\mathrm{C}$ is...; (3)if $\mathrm{A}$ or $\mathrm{B}$ and $\mathrm{C}$ or $\mathrm{D}$ is ... then $\mathrm{E}$ is.... where (1) and (2) A and B are joint of a robot arm input variables parameter, also known as the premise variables; $\mathrm{C}$ is the conclusion parameters; and (3) A, B, C and D are joint of a robot arm input variables parameter, also known as the premise variables, $\mathrm{E}$ is the conclusion parameters.

For the same input parameters, through the selection of different design rules, so as to reduce the output quantization error, so that the output closer to the input, and then up to maturity.

The Robot Arm Trajectory Planning. In 1995, Denavit and Hartenber have proposed the D-H coordinate system, and later developed into a standard method for modeling the motion of the robot [9-10]. Using D-H notation to model the robot, we first need to specify a local reference coordinate system for each joint. D-H coordinates of robot arm with 6 degrees of freedom in the two joints as shown in Fig. 3. The rest joint coordinates are established in that way.

Table 1 is robot parameters for experiments. By analyzing the parameters of the robot, the transformation matrix can be calculated by formula (1). 
By using the D-H method to get the matrix transformation, robot armwith 6 degrees of freedom rotation angle can be calculated, and the robot arm trajectory can be calculated from the angle of rotation, which can achieve the desired objectives.

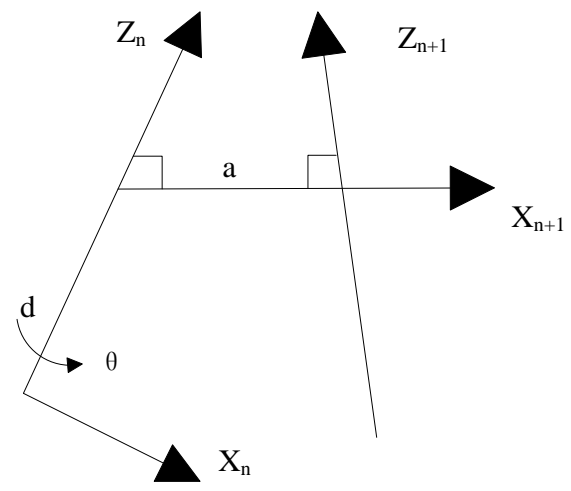

(a) $a$ joint

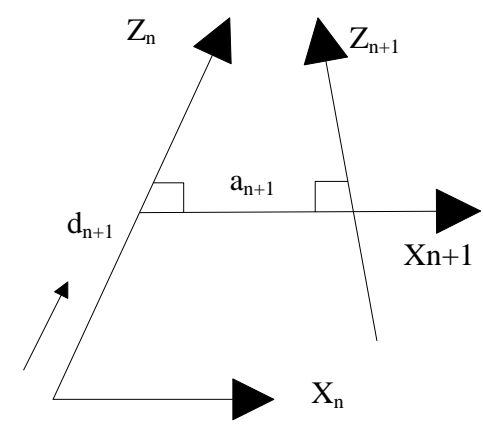

(b) $a_{\mathrm{n}+1}$ joint

Figure 3. D-H coordinates of robot arm with 6 degrees of freedom in the two joints

Table 1 Parameters of Robot Arm Joint

\begin{tabular}{|c|c|c|c|c|}
\hline$\#$ & $\boldsymbol{\theta}$ & $\boldsymbol{d}$ & $\boldsymbol{a}$ & $\boldsymbol{\alpha}$ \\
\hline 1 & $\theta_{1}$ & 0 & 0 & 90 \\
\hline 2 & $\theta_{2}$ & 0 & $\mathrm{a}_{2}$ & 0 \\
\hline 3 & $\theta_{3}$ & 0 & $\mathrm{a}_{3}$ & -90 \\
\hline 4 & $\theta_{4}$ & 0 & $\mathrm{a}_{4}$ & 90 \\
\hline 5 & $\theta_{5}$ & 0 & 0 & 0 \\
\hline 6 & $\theta_{6}$ & 0 & 0 & 0 \\
\hline
\end{tabular}

Where $\boldsymbol{\theta}$ is around $\mathrm{Z}$ axis rotation angle, $d$ is said sliding joint variables (because the robot joint is rotating, so all joint variables are angle), $\boldsymbol{a}$ denotes the $Z_{n}$ and $Z_{n+1}$ between the common vertical line, $\alpha$ around the $\mathrm{X}$ axis rotation angle.

$A_{1}=\left[\begin{array}{ccccc}\cos \theta_{1} & 0 & \sin \theta_{1} & 0 \\ \sin \theta_{1} & 0 & -\cos \theta_{1} & 0 \\ 0 & 1 & 0 & 0 \\ 0 & 0 & 0 & 1\end{array}\right] A_{2}=\left[\begin{array}{cccc}\cos \theta_{2} & -\sin \theta_{2} & 0 & \cos \theta_{2} \\ \sin \theta_{2} & \cos \theta_{2} & 0 & \sin \theta_{2} \\ 0 & 0 & 1 & 0 \\ 0 & 0 & 0 & 1\end{array}\right] A_{3}=\left[\begin{array}{cccc}\cos \theta_{3} & -\sin \theta_{3} & 0 & \cos \theta_{3} \\ \sin \theta_{3} & \cos \theta_{3} & 0 & \sin \theta_{3} \\ 0 & 0 & 0 & 1 \\ 0 & 0 & 0 & 1\end{array}\right]$ $A_{4}=\left[\begin{array}{cccc}\cos \theta_{4} & 0 & -\sin \theta_{4} & \cos \theta_{4} \\ \sin \theta_{4} & 0 & \cos \theta_{4} & \sin \theta_{4} \\ 0 & -1 & 0 & 0 \\ 0 & 0 & 0 & 1\end{array}\right] A_{5}=\left[\begin{array}{cccc}\cos \theta_{5} & 0 & \sin \theta_{5} & 0 \\ \sin \theta_{5} & 0 & -\cos \theta_{5} & 0 \\ 0 & 1 & 0 & 0 \\ 0 & 0 & 0 & 1\end{array}\right] A_{6}=\left[\begin{array}{cccc}\cos \theta_{6} & -\sin \theta_{6} & 0 & 0 \\ \sin \theta_{6} & \cos \theta_{6} & 0 & 0 \\ 0 & 0 & 1 & 0 \\ 0 & 0 & 0 & 1\end{array}\right]$

\section{Design of Control System for Robot Arm with 6 Degrees of Freedom}

Software Design. The matrix-based MATLAB language is the world's most natural way to express computational mathematics. Built-in graphics make it easy to visualize and gain insights from data. A vast library of prebuilt toolboxes lets you get started right away with algorithms essential to your domain. The desktop environment invites experimentation, exploration, and discovery. These MATLAB tools and capabilities are all rigorously tested and designed to work together. In this way, technical's work becomes simple and efficient. Simulation software of adaptive fuzzy control provides 
a good interface for teaching and engineering people, which makes the building of the system model become more easier than before.

Through the structure of fuzzy control simulation software, we can see that the MATLAB's can deal with the system simulation and data processing. System emulation with MATLAB's circumstance, robot's toolbox , MALAB's language and the parameters of robot arm joint. Also, don't forget to write the corresponding code. Degrees of freedom robot arm system simulation diagram is as Fig. 4. We can visually see the 3D simulation results from the simulation diagram .

We can find out the robot kinematics solution of the problem with Robotics Toolbox in the MATLAB's circumstance. One of the robot's joints is shown in Fig. 5. Through the graph, we can know that the motion curve of robot's is the same with the predicted results.
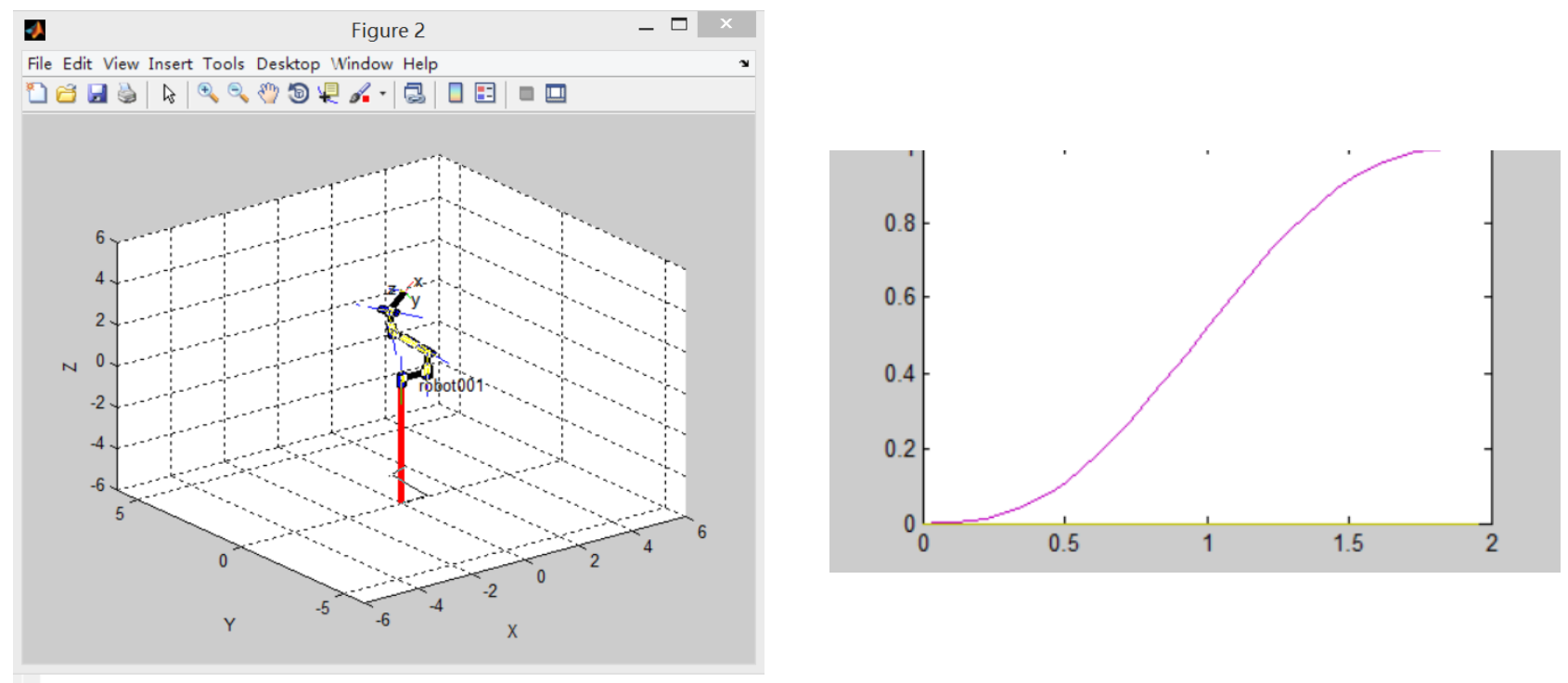

Figure 4. Simulation Diagram Robot Arm with Six Figure 5. Graph of One of the robot's joints Degrees of Freedom

Arduino Platform. Control system's design is based on the Arduino board of Arduino platform, which includes Atmel's microprocessors and circuit board. Software program can edit and compile in the Arduino IDE's environment, using USB interface can be uploaded to the Arduino control board, after that, the program control hardware successfully.

Arduino board is an open source hardware platform with AVR single chip microcomputer as the core, its open nature makes operation more convenient, at the same time its internal and bring their own software, thus is advantageous to the secondary development use.

ATmega168 is enhanced based on AVR RISC structure of low-power 8-bit CMOS microcontroller. Because of its advanced instruction set and clock cycle instruction execution time, ATmega168 data throughput rate is as high as $1 \mathrm{MIPS} / \mathrm{MHz}$, which can ease the contradiction between in terms of power and speed. In addition, it also provides a nonvolatile memory chips: $16 \mathrm{k}$ Bytes Flash, $1 \mathrm{k}$ Bytes SRAM block and 512 Bytes EEPROM. Chip article has 32 GPIO lines, which includes a programmable USART serial interface, $\mathrm{I}^{2} \mathrm{C}$ serial interface and two line SPI serial interface, etc.

\section{Experiment}

Communication between Arduino and PC. Based on Arduino platform, using the PC machine to control the steering engine to achieve the control of the robot arm. In order to more intuitive and simple debugging, using the "host computer+Arduino+servo controller" open system structure, the system debugging block diagram as shown in Fig. 6. 


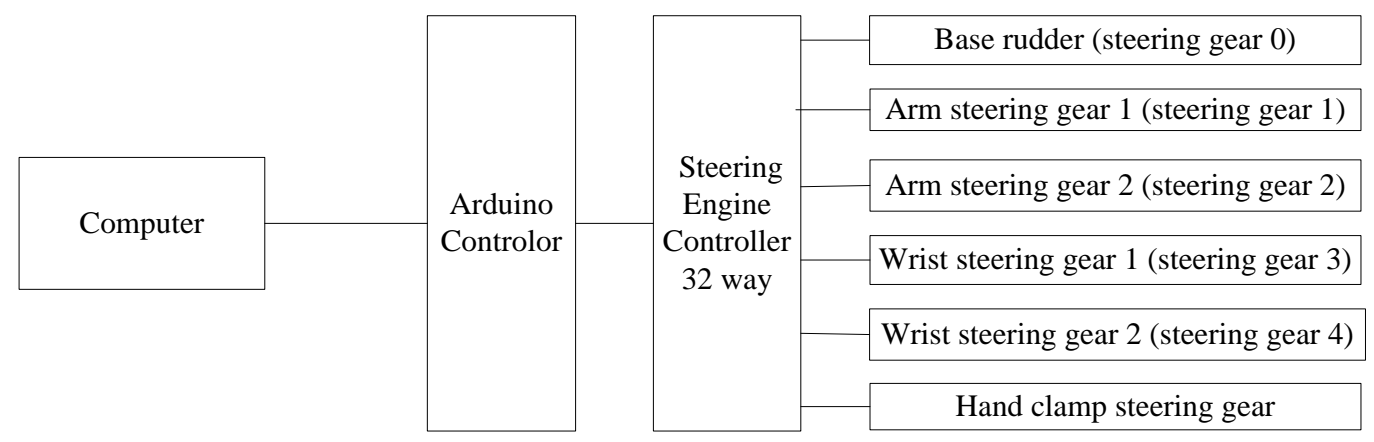

Figure 6. Block Diagram of system debugging

In the experiment, the use of Arduino control board, in which the host computer and Arduino control panel through the USB serial communication. Using USB to communicate with the use of convenient, fast and flexible connection and other features, only the use of a USB data line, you can connect the PC and Arduino control board.

On Line Debugging of Arduino and PC. The PC software, and through the control the Arduino board corresponding I/O port to control on the steering gear, reach the purpose of each joint of the robot control, realize PC and Arduino.

\section{Summary}

This paper begins with a discussion of the various adaptive control methods, according to various control law for the proposed adaptive fuzzy control method as the research method of this thesis.

It then discusses the use to achieve robot arm with 6 degree of freedom simulation in MATLAB in the Arduino platform hardware and software development. The developed software platform can be used for teaching and scientific research, as well as the interface, which can be used for further development.

Finally, a variety of serial communication methods and the use of PC software and Arduino control board on robot arm.

\section{Acknowledgement}

This work was financially supported by the National Natural Science Foundation of China (Grant No. 6154055), the Doctor's scientific research foundation of Hezhou University (No.HZUBS201506).

\section{References}

[1] Amitava Chatterjee and Ranajit Chatterjee, "Augmented Stable Fuzzy Control for Flexible Robotic Arm Using LMI Approach and Neuro-Fuzzy State Space Modeling," IEEE Trans. on Industrial Electronics, vol. 55, no, 3, pp. 3671-3681, March 2008.

[2] W. G. Hao, Y. Y. Leck, and L. C. Hun," 6-DOF PC-based Robotic Arm (PC-ROBOARM) with efficient trajectory planning and speed control" in Proc. of the International Conference on Mechatronics (ICOM), Kuala Lumpur, Malaysia, 17-19 May 2011.

[3] R. J. Wang, J. W. Zhang, J. M. Xu, and H. Y. Liu, "The Multiple-Function Intelligent Robotic Arms," in Proc. of the IEEE International Conference on Fuzzy Systems, Korea, August 20-24, 2009.

[4] Hideyuki Uehara, Hiroki Higa, and Takashi Soken, "A Mobile Robotic Arm for People with Severe Disabilities," in Proc. of the International Conference on Biomedical Robotics and Biomechatronics, Tokyo, Japan, September 26-29, 2010. 
[5] Roland Szabó and Ioan Lie, "Automated Colored Object Sorting Application for Robotic Arms," International Symposium on Electronics and Telecommunications, Timisoara, 2012.

[6] M. Nkomo and M. Collier, "A Color-Sorting SCARA Robotic Arm" in Proc. Of the International Conference on Consumer Electronics, Yichang, China, April 2012, pp. 763-768.

[7] G. Feng, S. G. Cao, N. W. Rees, and C. K. Chak, "Design of fuzzy control systems with guaranteed stability," Fuzzy Sets Syst., vol. 85, no. 1, pp. 1-10, January 1997.

[8] S. G. Cao, N. W. Rees, and G. Feng, "Stability analysis and design for a class of continuous-time fuzzy control systems,” Int. J. Control, vol. 64, no. 6, pp. 1069-1087, August 1996.

[9] X. S. Jiang, "Introduction to Robotics," Liaoning Science and Technology Press, Shenyang, 1994.

[10]Z. X. Cai, “The Robotics,” Tsinghua University Press, Beijing, 2000. 